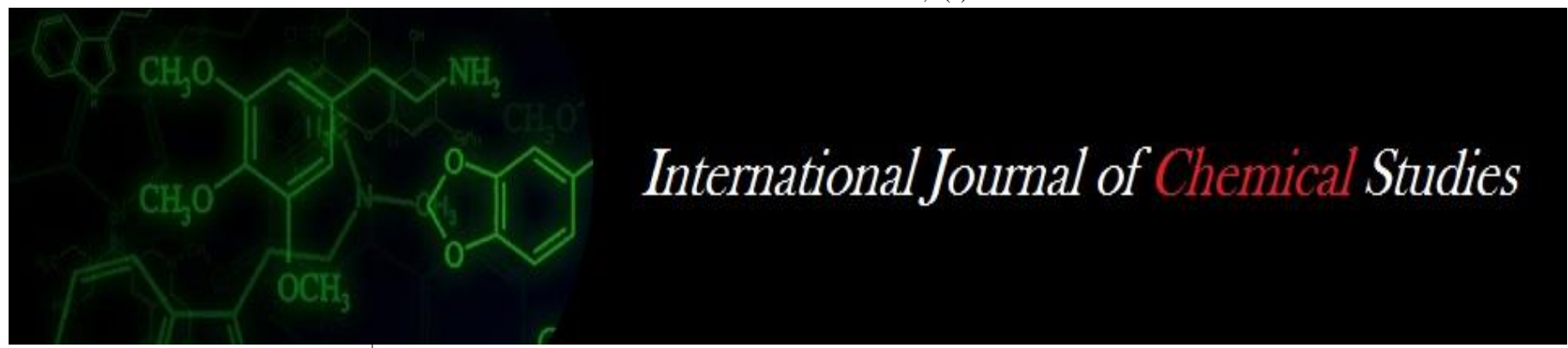

P-ISSN: 2349-8528

E-ISSN: 2321-4902

www.chemijournal.com

IJCS 2020; 8(6): 344-349

(C) 2020 IJCS

Received: 25-09-2020

Accepted: 27-10-2020

\section{B Meena}

Regional Research Station, Tamil Nadu Agricultural

University, Vridhachalam,

Cuddalore District, Tamil Nadu, India
Corresponding Author:

\section{B Meena}

Regional Research Station,

Tamil Nadu Agricultural

University, Vridhachalam,

Cuddalore District, Tamil Nadu

India

\section{Identification of resistant sources in sesame germplasm to major diseases}

\section{B Meena}

DOI: https://doi.org/10.22271/chemi.2020.v8.i6e.10792

Abstract

Root rot caused by Macrophomina phaseolina (Tassi) Goid is the destructive disease in sesame. Foliar diseases viz., phyllody, Alternaria leaf blight and powdery mildew cause major threat in sesame cultivation. About two hundred sesame germplasm were screened for major diseases viz., root rot, phyllody, Alternaria leaf blight and powdery mildew under field conditions. The sesame germplasm viz., SI-2116 (18.4\%), SIC-17326 (18.2\%) and IS-351-2 (19.7\%) were found to be moderately resistant to root rot disease. None of the germplasm recorded less than $10 \%$ of root rot disease incidence among the 200 germplasms screened. The maximum root rot disease incidence of $64.8 \%$ was observed in the susceptible check VRI -1(64.8\%). Phyllody disease was found to be the lowest of $5.7 \%$ in sesame germplasm viz., EC-303440-B. Powdery mildew disease ranged from 0 to 2 grade and Alternaria leaf spot disease was observed in the range of 1 to 3 grade.

Keywords: Sesame, germplasm, screening, diseases

\section{Introduction}

Sesame seed is a rich source of protein (20\%), edible oil (50\%), oleic acid (47\%) and linolenic acid $(39 \%)$. Although sesame is widely used for different purposes, it has low productivity due to non-availability of high-yielding varieties, resistant variety to biotic and abiotic stresses, low harvest index, seed shattering and indeterminate growth habit (Vyas et al., 1984) ${ }^{[10]}$. The low productivity of sesame has been attributed to pests and disease occurrence (Buldeo and Rane, 1978) ${ }^{[1]}$. Many diseases attack sesame, but only a few of them such as Fusarium wilt, charcoal rot, stem and root rot, bacterial blight, bacterial leaf spot, Cercospora leaf spot, Alternaria leaf spot, powdery mildew, leaf curl and phyllody are considered to be important diseases of sesame in the world and it occurs wherever sesame is cultivated. Among the diseases, root rot caused by Macrophomina phaseolina (Tassi) Goid is the most serious one affecting the crop at the later stages of growth. Maiti et al. (1988) ${ }^{[6]}$ reported an estimated yield loss of $57 \%$ at about $40 \%$ of disease incidence. The most common symptom of the disease is the sudden wilting of growing plants mainly after the flowering stage, the stem and roots become black due to severe infection. High temperature and water stress during growing season favours the pathogen's incidence (Chattopadhyay and Kalpana Sastry, 1998) [2]. Association of phytoplasma has been confirmed with phyllody disease in India on the basis of symptoms, electron microscopy and molecular approaches but only up to group level the incidence of phyllody disease increased day by day in sesame growing areas. Phyllody is associated with a mycoplasma-like organism (MLO) in the phloem of affected plants. It is transmitted by leafhopper. Singh (1987) ${ }^{[9]}$ reported 5-85 per cent yield losses due to Alternaria leaf spot disease.

Hence, the present study was conducted to assess the extent of damage caused by various diseases in sesame germplasm in order to identify the resistant sources for major diseases.

\section{Materials and Methods}

Sesame (Sesamum indicum L.) plants showing typical root rot symptoms were collected and the isolation of fungus was done following the standard tissue isolation technique. Those parts of root and stem showing typical symptoms of the disease were washed in running tap water and cut into small bits. These bits were surface sterilized with 0.1 per cent mercuric chloride 
solutions for 30 seconds and washed thoroughly in sterile distilled water for three times to remove traces of mercuric chloride and then aseptically transferred to sterilized potato dextrose agar (PDA) plates and incubated at $27 \pm 1^{\circ} \mathrm{C}$ for three days for fungal growth. Later, the bit of fungal growth was transferred to PDA slants. The pure culture of the fungus was obtained by further growing the culture under aseptic conditions by following hyphal tip culture method (Rangaswami, 1972) ${ }^{[7]}$. After seven days of incubation, pure isolates were obtained and maintained at $4^{\circ} \mathrm{C}$ for further studies.

The pathogenic ability of M. phaseolina (isolated from the diseased stem) was tested in screen house on sesame. Culture of $M$. phaseolina was raised in $250 \mathrm{ml}$ Erlenmeyer flask containing $50 \mathrm{ml}$ of PDB (potato dextrose broth) sterilized at $15 \mathrm{lbs}$ per sq inch pressure for 20 minutes. The bits of $5 \mathrm{~mm}$ size were cut with the help of sterilized cork borer from fresh pure culture plates ( 5 days old) and transferred into flasks with the help of sterilized needle under aseptic conditions. After seven days of incubation in BOD incubator at $27 \pm 1^{\circ} \mathrm{C}$, mycelial mats were collected and dried between folds of blotting paper for further use. Five gram of fresh mycelial mat was homogenized in blender for 2 minutes at lowest speed in $1000 \mathrm{ml}$ of sterilized water. The suspension was used to inoculate the pots containing $5 \mathrm{~kg}$ of sand: ground sesame seed mixture (9:1) which was sterilized by autoclaving at 15 psi for one and half hours for two consecutive days. On the third day of inoculation, thirty seeds of sesame were sown in pots. Pots were irrigated regularly to maintain moisture. After 8-10 days of sowing, the symptoms appeared and the infected plants exhibited elongated lesions at collar region which will be later converted to dark brown to black and stem was completely girdled by the lesions. The affected plants wilted and dried up later. Diseased plants were brought to laboratory and isolations were made on PDA medium from diseased stem to confirm the identity of pathogen (Riker and Riker, 1936) ${ }^{[8]}$.

\section{Evaluation of sesame germplasm for resistance to major diseases}

The field trial was conducted at New farm, Regional Research Station, Vridhachalam during kharif 2018 under sick plot conditions created for root rot pathogen, Macrophomina phaseolina. About two hundred sesame germplasm along with the susceptible check VRI -1were screened against major diseases of sesame under field conditions in Randomized block design in two rows of $3 \mathrm{~m}$ length and replicated thrice. In addition to root rot disease, other diseases viz., phyllody, Alternaria leaf blight and powdery mildew disease incidences were recorded under field conditions. The root rot disease and phyllody disease incidence were recorded at 70 days after sowing by counting the number of diseased plants and total plants. Alternaria leaf blight and powdery mildew disease intensity was recorded using the grade $0-5$ scale. The sesame germplasm were graded as resistant, moderately resistant, moderately susceptible, susceptible or highly susceptible based on their infection percentage using the scale given by Dinakaran and Naina Mohammed (2001) ${ }^{[3]}$.

\begin{tabular}{|c|c|c|}
\hline Disease scale & Per cent infection (\%) & Reaction \\
\hline 1 & $1-10$ & Resistant \\
\hline 3 & $11-20$ & Moderately resistant \\
\hline 5 & $21-30$ & Moderately susceptible \\
\hline 7 & $31-50$ & Susceptible \\
\hline 9 & $51-100$ & Highly susceptible \\
\hline
\end{tabular}

The reaction of sesame genotypes to diseases was assessed and the results were furnished in Table 1.

\section{Results and Discussion}

In the present study, two hundred sesame germplasm were evaluated along with the susceptible check VRI-1 against major diseases. Three types of disease response i.e., moderately resistant, moderately susceptible and susceptible reactions were observed in the present study. Among the 200 sesame germplasm lines screened under sick plot conditions, disease incidence of root rot ranged from $18.2 \%$ (SIC-17326) to $68.4 \%$ (KMR-1). The lowest root rot disease incidence of $18.2 \%$ was recorded in the sesame germplasm viz., SIC17326. The maximum root rot disease incidence was observed in the sesame germplasm viz., KMR-1. From the results it was found that the sesame germplasm viz., SI-2116 (18.4\%), SIC$17326(18.2 \%)$ and IS-351-2 (19.7\%) were found to be moderately resistant to root rot disease (Table 1).

The sesame germplasm viz., ICA-14146-A (21.3\%), IS-100-8 (24.6\%), SI-76-1(21.4\%), EC-335010 (24.8\%), EC-335010 (21.4\%), SI-1033 (20.4\%), EC-33962 (24.6\%), SI-253 (21.8\%), ES-75-2-84 (24.2), IS-461-1-84-I (24.8\%), NIC$163-88$ (21.2\%), GSM-22 (24.7\%), S-0308 (22.6\%), GRT83135 (24.7\%), IS-355 (20.6\%), SI-3218 (24.7\%), MIC-8526 (22.8\%), EC-310421 (20.6\%), NAL/28/27/31/4 (24.7\%), KMR-77-I (20.3\%), B-203 (21.7\%), S-0337 (21.3\%), NIC16236 (20.4\%), EC-303445 (21.6\%), NIC-8533 (22.4\%), IC$96113(22.3 \%)$, GRT-8339 (21.4\%), IC-14120-1 (22.7\%), NIC-8165 (21.7\%), ES-29 (22.8\%), S-0439 (21.9\%), IC131485 (20.4\%), IC-204595 (21.7\%), NIC-10622 (21.8\%), SI-1818 (22.4\%) ES-150-1 (21.4\%), SI-2952 (21.6\%), KMR$27(21.2 \%)$, ES-20 (21.4\%), KMR-10 (22.8\%), KMR-22 (21.6\%), IS-199 (21.7\%), S-0534 (22.8\%), IS-201 (21.4\%), KM-86 (22.7\%) and ES-110 (21.4\%) recorded root rot disease incidence from 20.0 to $25.0 \%$ (Moderately susceptible). From the screening results, it was found that rest of the lines were found susceptible to root rot disease (Table 1).

The sesame germplasm viz., SI-3179, SI-3231, EC-335011-A, IS-665, EC-334984, EC-334001, IS-461-1-84, EC-334995, EC-3349997, KMR-1, IS-17, IS-722-2-84, IS-3179, IS-393-1, SI-995, SI-2008, NIC-8288, S-0228, S-022, GRT-8359-I and KMS-322-I recorded maximum root rot disease incidence at the time of physiological maturity. None of the germplasm recorded less than $10 \%$ of root rot disease incidence among the 200 germplasms screened. The maximum root rot disease incidence of $64.8 \%$ was observed in the susceptible check VRI -1(64.8\%). Phyllody incidence ranged from 5.7\% (EC$303440-B)$ to $18.4 . \%$ (SI-3237) among the sesame germplasm screened. The susceptible check VRI -1recorded the maximum phyllody disease incidence of $23.6 \%$. Powdery mildew disease incidence ranged from 0 to 2 grade among the 200 sesame germplasms screened. In the screening of sesame germplasm, Alternaria leaf spot incidence was observed in the range between 1 to 3 grade (Table 1).

The sesame germplasm viz., SI-1156(8.7\%), EC-335011$\mathrm{A}(9.4 \%)$, BC-303427(7.9\%), IS-665(6.4\%), SI-3234(6.2\%), IS-475(9.3\%), IS-100-8(7.6\%), SI-1679(8.3\%), SI-761(7.4\%), EC-334984(7.2\%), SP-1144(8.3\%), EC334001(9.7\%), KIS-398(8.4\%), KIS-398(7.6\%), EC334973(8.1\%), EC-178-2(8.4\%) recorded lesser phyllody disease incidence (Table 1).

Identification of disease resistant lines is a major goal for plant breeders. Breeding for disease resistance requires efficient, low-cost and rapid screening techniques (Foolad et $a l ., 2000)^{[5]}$. In the present study, there was no perfect 
resistance was observed although germplasm having perfect resistance (without symptom) to wilt disease was reported previously (El-Shazly et al., 1999) ${ }^{[4]}$. However, the identified moderately resistant lines may be utilized for breeding programmes to broaden the resistance against major diseases of sesame.

Table 1: Screening of sesame germplasm against major diseases

\begin{tabular}{|c|c|c|c|c|c|}
\hline S. No. & Germplasm & Root $\operatorname{rot}(\%)$ & Phyllody (\%) & Alternaria leaf spot (0-5 scale) & Powdery mildew (0-5 scale) \\
\hline 1 & SI-3237 & 36.2 & $18 . .4$ & 2 & 1 \\
\hline 2 & IC-131607 & 44.6 & 17.6 & 3 & 2 \\
\hline 3 & SI-3179 & 56.3 & 14.7 & 3 & 1 \\
\hline 4 & SI-3231 & 60.4 & 13.9 & 2 & 0 \\
\hline 5 & EC-33507 & 54.8 & 14.2 & 1 & 2 \\
\hline 6 & IS-321 & 48.6 & 16.3 & 2 & 1 \\
\hline 7 & SI-1156 & 20.3 & 8.7 & 1 & 0 \\
\hline 8 & EC-335011-A & 57.2 & 9.4 & 2 & 1 \\
\hline 9 & EC-334990 & 28.4 & 12.3 & 1 & 0 \\
\hline 10 & EC-334989 & 26.7 & 11.6 & 1 & 0 \\
\hline 11 & ICA-14146-A & 21.3 & 13.4 & 2 & 1 \\
\hline 12 & BC-303427 & 3.6 & 7.9 & 2 & 2 \\
\hline 13 & IS-665 & 56.9 & 6.4 & 3 & 1 \\
\hline 14 & SI-3234 & 51.4 & 6.2 & 2 & 1 \\
\hline 15 & EC-334280 & 44.3 & 10.6 & 3 & 2 \\
\hline 16 & S-0182-I & 48.6 & 11.4 & 2 & 0 \\
\hline 17 & IS-475 & 40.3 & 9.3 & 2 & 1 \\
\hline 18 & EC-334983 & 45.8 & 12.6 & 1 & 1 \\
\hline 19 & KIS-375 & 48.7 & 10.4 & 3 & 2 \\
\hline 20 & Agra-balik & 54.3 & 11.3 & 2 & 0 \\
\hline 21 & IS-100-8 & 24.6 & 7.6 & 1 & 2 \\
\hline 22 & SI-1679 & 36.7 & 8.3 & 1 & 0 \\
\hline 23 & SI-76-1 & 21.4 & 7.4 & 1 & 1 \\
\hline 24 & EC-334984 & 66.2 & 7.2 & 2 & 1 \\
\hline 25 & SP-1144 & 32.6 & 8.3 & 3 & 2 \\
\hline 26 & EC-334950-I & 36.4 & 10.4 & 2 & 1 \\
\hline 27 & EC-335010 & 24.8 & 11.3 & 1 & 1 \\
\hline 28 & EC-334001 & 57.1 & 9.7 & 3 & 0 \\
\hline 29 & EC-334979 & 48.4 & 10.6 & 2 & 1 \\
\hline 30 & KIS-398 & 51.6 & 8.4 & 3 & 2 \\
\hline 31 & EC-334977 & 54.9 & 7.6 & 2 & 2 \\
\hline 32 & KIC-1634-B & 36.6 & 10.8 & 1 & 1 \\
\hline 33 & EC-334973 & 39.8 & 8.1 & 2 & 1 \\
\hline 34 & EC-178-2 & 48.2 & 8.4 & 2 & 2 \\
\hline 35 & SI-1516 & 42.7 & 10.3 & 1 & 1 \\
\hline 36 & IS-728 & 39.6 & 7.8 & 2 & 1 \\
\hline 37 & EC-334985-I & 44.2 & 7.2 & 3 & 2 \\
\hline 38 & EC-334994 & 21.4 & 6.7 & 1 & 2 \\
\hline 39 & EC-334974 & 45.7 & 8.3 & 2 & 1 \\
\hline 40 & SI-349 & 54.6 & 10.6 & 2 & 1 \\
\hline 41 & IS-461-1-84 & 63.2 & 9.4 & 3 & 0 \\
\hline 42 & EC-334999 & 39.8 & 8.1 & 2 & 2 \\
\hline 43 & NIC-7905 & 28.3 & 9.2 & 1 & 1 \\
\hline 44 & SI-1687 & 42.5 & 7.6 & 2 & 1 \\
\hline 45 & EC-3340998 & 33.8 & 9.7 & 1 & 2 \\
\hline 46 & SI-1033 & 20.4 & 11.3 & 1 & 2 \\
\hline 47 & EC-665 & 26.2 & 8.6 & 1 & $\frac{2}{1}$ \\
\hline 48 & SI-1225 & 36.7 & 9.7 & 2 & 0 \\
\hline 49 & IS-366 & 58.2 & 8.3 & 3 & 1 \\
\hline 50 & EC-33962 & 24.6 & 10.6 & 1 & 2 \\
\hline 51 & IS-723 & 26.3 & 9.7 & 1 & 0 \\
\hline 52 & SI-253 & 21.8 & 10.4 & 2 & 0 \\
\hline 53 & S-0388 & 38.7 & 8.7 & 2 & 1 \\
\hline 54 & ES-75-2-84 & 24.2 & 10.2 & 1 & 1 \\
\hline 55 & ES-334966 & 28.4 & 8.3 & 1 & 2 \\
\hline 56 & ES-81 & 51.7 & 7.4 & 3 & 0 \\
\hline 57 & IC-199443 & 42.4 & 9.2 & 2 & 1 \\
\hline 58 & EC-334995 & 62.6 & 8.6 & 2 & 2 \\
\hline 59 & EC-3349997 & 64.1 & 10.3 & 1 & 2 \\
\hline 60 & KMR-1 & 68.4 & 9.7 & 2 & 1 \\
\hline 61 & ES-62 & 48.3 & 13.4 & 2 & 1 \\
\hline 62 & SI-2192 & 36.7 & 16.2 & 3 & 2 \\
\hline
\end{tabular}




\begin{tabular}{|c|c|c|c|c|c|}
\hline 63 & IS-17 & 64.9 & 15.7 & 2 & 1 \\
\hline 64 & IS-722-2-84 & 62.3 & 11.3 & 2 & 2 \\
\hline 65 & IS-3179 & 61.8 & 9.7 & 2 & 1 \\
\hline 66 & IS-446-1-64 & 28.6 & 12.4 & 1 & 2 \\
\hline 67 & IS-393-1 & 68.3 & 13.7 & 3 & 2 \\
\hline 68 & EC-303440-B & 42.6 & 5.7 & 2 & 2 \\
\hline 69 & IS-461-1-84-I & 24.8 & 8.6 & 1 & 1 \\
\hline 70 & EC-335005 & 27.3 & 7.4 & 2 & 1 \\
\hline 71 & NIC-163-88 & 21.2 & 6.9 & 2 & 2 \\
\hline 72 & SI-995 & 63.8 & 7.3 & 3 & 1 \\
\hline 73 & SI-1345 & 36.4 & 12.7 & 2 & 2 \\
\hline 74 & SI-63 & 45.1 & 13.6 & 2 & 1 \\
\hline 75 & EC-334993 & 28.8 & 16.4 & 1 & 2 \\
\hline 76 & SI-2008 & 60.7 & 11.7 & 2 & 0 \\
\hline 77 & NIC-8288 & 62.4 & 13.4 & 2 & 1 \\
\hline 78 & EC-334971 & 48.3 & 10.6 & 1 & 2 \\
\hline 79 & EC-310439 & 54.2 & 11.7 & 2 & 2 \\
\hline 80 & SI-7192 & 56.4 & 8.6 & 3 & 1 \\
\hline 81 & SI-3070 & 34.6 & 10.4 & 1 & 1 \\
\hline 82 & GSM-22 & 24.7 & 12.6 & 1 & 2 \\
\hline 83 & SI-2973 & 38.2 & 9.7 & 2 & 1 \\
\hline 84 & S-0308 & 22.6 & 12.4 & 1 & 1 \\
\hline 85 & NIC-16328 & 26.8 & 8.6 & 2 & 2 \\
\hline 86 & GRT-83135 & 24.7 & 9.7 & 2 & 0 \\
\hline 87 & GRT-83125 & 29.3 & 13.4 & 2 & 2 \\
\hline 88 & IS-56 & 39.6 & 11.7 & 3 & 1 \\
\hline 89 & NIC-16268 & 27.4 & 9.4 & 2 & 1 \\
\hline 90 & NIC-16275 & 48.6 & 10.3 & 2 & 2 \\
\hline 91 & NIC-8984 & 33.2 & 14.6 & 1 & 0 \\
\hline 92 & MT-67-25 & 28.1 & 11.7 & 1 & 1 \\
\hline 93 & IS-355 & 20.6 & 16.2 & 2 & 0 \\
\hline 94 & SI-3218 & 24.7 & 9.4 & 2 & 2 \\
\hline 95 & MIC-8526 & 22.8 & 10.3 & 2 & 1 \\
\hline 96 & SI-2116 & 18.4 & 13.4 & 1 & 2 \\
\hline 97 & SIC-17326 & 18.2 & 9.7 & 1 & 1 \\
\hline 98 & EC-310421 & 20.6 & 13.6 & 1 & 0 \\
\hline 99 & S-0335 & 27.8 & 11.4 & 2 & 1 \\
\hline 100 & NAL/28/27/31/4 & 24.7 & 10.7 & 2 & 2 \\
\hline 101 & IC-14093 & 32.4 & 14.9 & 2 & 1 \\
\hline 102 & KMR-77-I & 20.3 & 7.3 & 1 & 0 \\
\hline 103 & IS-351-2 & 19.7 & 9.7 & 2 & 1 \\
\hline 104 & S-0448 & 32.6 & 17.6 & 3 & 2 \\
\hline 105 & KIS-375-I & 24.3 & 12.3 & 2 & 1 \\
\hline 106 & GRT-83148 & 26.9 & 14.4 & 1 & 0 \\
\hline 107 & B-203 & 21.7 & 9.7 & 1 & 1 \\
\hline 108 & IC-14160-I & 24.8 & 10.3 & 2 & 2 \\
\hline 109 & S-0337 & 21.3 & 8.6 & 1 & 1 \\
\hline 110 & KMR-17 & 39.6 & 9.4 & 2 & 2 \\
\hline 111 & IC-96128 & 54.8 & 11.3 & 3 & 2 \\
\hline 112 & S-0228 & 57.4 & 9.7 & 2 & 1 \\
\hline 113 & S-0434 & 62.7 & 7.9 & 3 & 2 \\
\hline 114 & ES-110-C & 56.3 & 7.4 & 2 & 1 \\
\hline 115 & IS-607-1-84 & 54.9 & 9.6 & 2 & 0 \\
\hline 116 & NIC-16236 & 20.4 & 11.4 & 1 & 0 \\
\hline 117 & SI-1926 & 28.7 & 13.2 & 1 & 0 \\
\hline 118 & NIC-13598 & 32.6 & 9.7 & 2 & 2 \\
\hline 119 & IS-308-A & 36.5 & 16.3 & 2 & 1 \\
\hline 120 & MT-67-61 & 28.1 & 13.4 & 1 & 2 \\
\hline 121 & IS-37 & 36.8 & 10.3 & 3 & 1 \\
\hline 122 & $78-301$ & 29.3 & 9.7 & 2 & 1 \\
\hline 123 & S-0430 & 33.8 & 8.9 & 2 & 2 \\
\hline 124 & GRT-8359-I & 62.7 & 9.3 & 3 & 2 \\
\hline 125 & SI-2008 & 39.6 & 11.4 & 2 & 1 \\
\hline 126 & IS-309 & 29.4 & 10.7 & 2 & 1 \\
\hline 127 & SI-2940 & 38.7 & 9.4 & 3 & 2 \\
\hline 128 & IC-382-2 & 28.6 & 13.6 & 2 & 1 \\
\hline 129 & SI-1061 & 42.3 & 12.4 & 3 & 2 \\
\hline 130 & KMS-322-I & 56.4 & 11.7 & 3 & 2 \\
\hline 131 & KIS-219 & 26.7 & 10.9 & 1 & 0 \\
\hline
\end{tabular}




\begin{tabular}{|c|c|c|c|c|c|}
\hline 132 & EC-303445 & 21.6 & 8.6 & 1 & 1 \\
\hline 133 & NIC-8533 & 22.4 & 6.4 & 1 & 2 \\
\hline 134 & VCR/82/No/10/NS & 28.2 & 8.3 & 1 & 1 \\
\hline 135 & IC-96113 & 22.3 & 7.9 & 2 & 1 \\
\hline 136 & NIC-9839-I & 48.8 & 10.3 & 3 & 2 \\
\hline 137 & SI-3280-I & 42.6 & 9.2 & 2 & 1 \\
\hline 138 & GRT-8339 & 21.4 & 10.7 & 1 & 0 \\
\hline 139 & EC-303419 & 29.6 & 8.4 & 2 & 2 \\
\hline 140 & RJS-56 & 38.2 & 10.3 & 3 & 2 \\
\hline 141 & DSK-1 & 38.6 & 12.7 & 2 & 0 \\
\hline 142 & IC-14120-1 & 22.7 & 10.3 & 1 & 1 \\
\hline 143 & 49-E-SPS-6 & 32.6 & 9.6 & 3 & 2 \\
\hline 144 & ES-48 & 24.3 & 13.4 & 2 & 0 \\
\hline 145 & NIC-8165 & 21.7 & 11.7 & 2 & 1 \\
\hline 146 & ES-29 & 22.8 & 11.2 & 2 & 1 \\
\hline 147 & NIC-8559 & 36.4 & 8.6 & 3 & 2 \\
\hline 148 & EC-334950 & 23.6 & 12.7 & 1 & 0 \\
\hline 149 & S-0439 & 21.9 & 10.3 & 2 & 1 \\
\hline 150 & IC-131485 & 20.4 & 9.6 & 2 & 1 \\
\hline 151 & IC-204595 & 21.7 & 7.3 & 2 & 1 \\
\hline 152 & RJS-124 & 24.3 & 12.7 & 2 & 2 \\
\hline 153 & NIC-8202 & 33.6 & 9.6 & 3 & 2 \\
\hline 154 & NIC-10622 & 21.8 & 8.3 & 1 & 1 \\
\hline 155 & KMR-38 & 36.2 & 10.7 & 2 & 2 \\
\hline 156 & Kanpur local & 33.7 & 9.6 & 2 & 1 \\
\hline 157 & KMR-77 & 48.6 & 9.3 & 2 & 2 \\
\hline 158 & ICA-14105 & 34.8 & 7.4 & 2 & 1 \\
\hline 159 & GRT-83147 & 52.1 & 8.1 & 3 & 2 \\
\hline 160 & NIC-7913 & 32.6 & 9.6 & 2 & 1 \\
\hline 161 & IS-649 & 36.3 & 7.4 & 3 & 2 \\
\hline 162 & SI-1818 & 22.4 & 8.7 & 3 & 1 \\
\hline 163 & ES-249 & 48.6 & 9.3 & 1 & 2 \\
\hline 164 & ES-2186-2 & 36.2 & 11.6 & 2 & 1 \\
\hline 165 & NIC-8060 & 28.3 & 10.3 & 3 & 2 \\
\hline 166 & NIC-7943 & 32.4 & 7.9 & 2 & 0 \\
\hline 167 & ES-28 & 39.6 & 8.7 & 2 & 1 \\
\hline 168 & MT-6262 & 28.1 & 7.3 & 3 & 2 \\
\hline 169 & SI-3283 & 48.3 & 6.9 & 2 & 2 \\
\hline 170 & S-0130 & 26.7 & 7.2 & 1 & 1 \\
\hline 171 & ES-150-1 & 21.4 & 8.3 & 1 & 0 \\
\hline 172 & NIC-16347 & 23.6 & 6.7 & 2 & 1 \\
\hline 173 & SI-2174 & 54.3 & 6.1 & 2 & 2 \\
\hline 174 & NIC-16129 & 52.1 & 11.3 & 2 & 1 \\
\hline 175 & B-7-11 & 24.2 & 8.7 & 3 & 2 \\
\hline 176 & TS-261 & 28.6 & 10.6 & 2 & 2 \\
\hline 177 & RJS-193 & 36.1 & 7.4 & 1 & 0 \\
\hline 178 & NIC-16324 & 29.8 & 8.1 & 1 & 1 \\
\hline 179 & SI-2952 & 21.6 & 7.9 & 3 & 2 \\
\hline 180 & ES-40 & 28.4 & 10.3 & 2 & 0 \\
\hline 181 & KMR-27 & 21.2 & 9.4 & 2 & 1 \\
\hline 182 & KMR-112 & 24.3 & 8.3 & 2 & 2 \\
\hline 183 & ES-20 & 21.4 & 5.6 & 1 & 0 \\
\hline 184 & KMR-35 & 23.7 & 7.9 & 2 & 1 \\
\hline 185 & KMR-10 & 22.8 & 12.4 & 2 & 2 \\
\hline 186 & KMR-33 & 26.1 & 9.6 & 1 & 1 \\
\hline 187 & KMR-114 & 24.9 & 10.3 & 2 & 2 \\
\hline 188 & KMR-22 & 21.6 & 8.6 & 1 & 2 \\
\hline 189 & S-0579 & 24.9 & 9.3 & 2 & 1 \\
\hline 190 & KMS-04-262 & 23.2 & 7.4 & 2 & 1 \\
\hline 191 & IS-199 & 21.7 & 8.7 & 1 & 2 \\
\hline 192 & SI-3315-5 & 23.4 & 6.3 & 3 & 2 \\
\hline 193 & S-0534 & 22.8 & 10.6 & 2 & 0 \\
\hline 194 & KMS-4-235 & 23.7 & 13.1 & 2 & 2 \\
\hline 195 & KM-90 & 28.3 & 15.4 & 2 & 1 \\
\hline 196 & IS-653 & 42.6 & 7.9 & 3 & 2 \\
\hline 197 & IS-201 & 21.4 & 8.3 & 1 & 0 \\
\hline 198 & KM-76 & 36.2 & 12.4 & 2 & 1 \\
\hline 199 & KM-86 & 22.7 & 10.6 & 1 & 2 \\
\hline 200 & ES-110 & 21.4 & 8.3 & 1 & 1 \\
\hline
\end{tabular}




\begin{tabular}{|c|c|c|c|c|c|}
\hline 201 & Check VRI -1 & 64.8 & 23.6 & 3 & 2 \\
\hline & CD $(\mathrm{P}=0.05)$ & 3.1 & 3.8 & - & - \\
\hline
\end{tabular}

\section{Acknowledgements}

The financial support rendered by the ICAR-All India Coordinated Research Project on Sesame \& Niger in carrying out this research work is gratefully acknowledged.

\section{References}

1. Buldeo AM, Rane MS. Fusarium wilt of sesamum. J Maharashtra Agric Univ 1978;3:167-170.

2. Chattopadhyay C, Kalpana Sastry R. Important diseases of sesame and their management options. In: IPM systems in Agriculture, (Oilseeds), RK Upadhyay, KG Mukerjie, RL Rajak (eds.), Oxford and IBH Publ., New Delhi 1978-1998.

3. Dinakaran D, Naina Mohammed SE. Sesame and Safflower Newsl 2001;16:68-71.

4. EL-Shazly MS, Abdul Wahid OA, EL-Ashry MA, Ammar SM, EL-Bramawy MA. Evaluation of resistance to Fusarium wilt disease in sesame germplasm. International Journal of Pest Management 1999;45:207210.

5. Foolad MR, Ntahimpera N, Christ BJ, Lin GY. Comparison of field, greenhouse, and detached-leaflet evaluations of tomato germplasm for early blight resistance. Plant Disease 2000;84:967-972.

6. Maiti S, Hegde MR, Chattopadhyay SB. Handbook of Annual Oilseed Crops. Oxford and IBH Publ. Co. Pvt. Ltd., New Delhi 1988.

7. Rangaswami G. Diseases of crop plants in India. Prentice Hall of India Pvt. Ltd. New Delhi 1972, 520.

8. Riker AJ, Riker AS. Introduction to research on plant diseases. John. S. Swift, C.M.C., New York 1936, 117.

9. Singh SN. Response of chilli cultivars to Alternaria alternata and losses under field conditions. Farm Sci. J 1987;2:96-97.

10. Vyas SC, Kotwel T, Prasad KMV, Jain AC. Notes on seed-borne fungi of sesamum and their control. Seed Res 1984;12:93-94. 